\title{
Flavor and Quark Mass Dependence of QCD Thermodynamics
}

\author{
F. Karsch, E. Laermann, A. Peikert, Ch. Schmidt and S. Stickan * \\ Fakultät für Physik, Universität Bielefeld, D-33615 Bielefeld, Germany
}

\begin{abstract}
We calculate the transition temperature in 2 and 3-flavor QCD using improved gauge and staggered fermion actions on lattices with temporal extent $N_{\tau}=4$. We find $T_{c}=173(8) \mathrm{MeV}$ and 154 (8) $\mathrm{MeV}$ for $n_{f}=2$ and 3 , respectively. In the case of 3-flavor QCD we present evidence that the chiral critical point, i.e. the second order endpoint of the line of first order chiral phase transitions, belongs to the universality class of the $3 \mathrm{~d}$ Ising model.
\end{abstract}

\section{Introduction}

Determining the properties of strongly interacting matter at high temperature, the temperature at which the transition to the quark-gluon plasma phase takes place as well as other critical parameters, is one of the basic goals in lattice studies of finite-T QCD. Numerical calculations which have been performed during the last years have shown that the details of the transition strongly depend on the number of quark flavors $\left(n_{f}\right)$ as well as the value of e.g. the pseudo-scalar meson mass $\left(m_{\mathrm{PS}}\right)$ which is controlled by the bare quark masses $\left(m_{q}\right)$. Furthermore, it became evident that the moderate values of the lattice spacing $(a \sim 0.25 \mathrm{fm})$ used in finite-T calculations with dynamical fermions lead to sizeable cut-off effects; different discretization schemes used in the fermion sector, e.g. the standard staggered and Wilson fermion formulation, lead to significantly different results for $T_{c}$ [1]. Calculations with improved actions thus seem to be mandatory to obtain reliable quantitative results for $T_{c}$ and its dependence on $n_{f}$ as well as $m_{\mathrm{PS}}$.

We will report here on results for $n_{f}=2$ and 3 obtained with a Symanzik improved gauge action and a staggered fermion action with improved rotational invariance, $i$. e . the $\mathrm{p} 4$-action with fat 1 link terms. This action has been used previously by us in studies of the flavor dependence of the QCD thermodynamics [2]. Further details on the

\footnotetext{
*The work has been supported by the TMR network ERBFMRX-CT-970122 and the DFG under grant Ka 1198/4-1 .
}

action and the algorithm used for our simulations are given in [2]. For a discussion of recent results with improved Wilson fermions see Ref. 3].

\section{Flavor and quark mass dependence of $T_{c}$}

The transition temperature in QCD with dynamical fermions has been found to be significantly smaller than in the pure gauge sector. This is in accordance with intuitive pictures of the phase transition based for instance on the thermodynamics of bag or percolation models. With decreasing $m_{q}$ hadrons become lighter and it becomes easier to build up a sufficiently high particle density that can trigger a phase transition. For the same reason such models also suggest that $T_{c}$ decreases when $n_{f}$ and in turn the number of light pseudo-scalar mesons increases. This general picture is confirmed by the lattice results presented in the following.

We have calculated the pseudo-critical couplings, $\beta_{c}\left(m_{q}\right)$, for the transition to the QCD plasma phase on lattices of size $16^{3} \times 4$. Calculations have been performed for 2 and 3 -flavor QCD in a wide range of quark masses, $0.025 \leq m_{q} \leq$ 1.0. The smallest quark mass value corresponds to $m_{\mathrm{PS}} \simeq 350 \mathrm{MeV}$.

In order to set a scale for the transition temperature we have performed calculations of the light meson spectrum and the string tension ${ }^{2}$ at

\footnotetext{
${ }^{2}$ We note that the heavy quark potential is no longer strictly confining in the presence of dynamical quarks. The definition of the string tension is based on potentials extracted from Wilson loops which have been found to lead to a linear rising potential at least up to distances $r \sim 2 \mathrm{fm}$.
} 

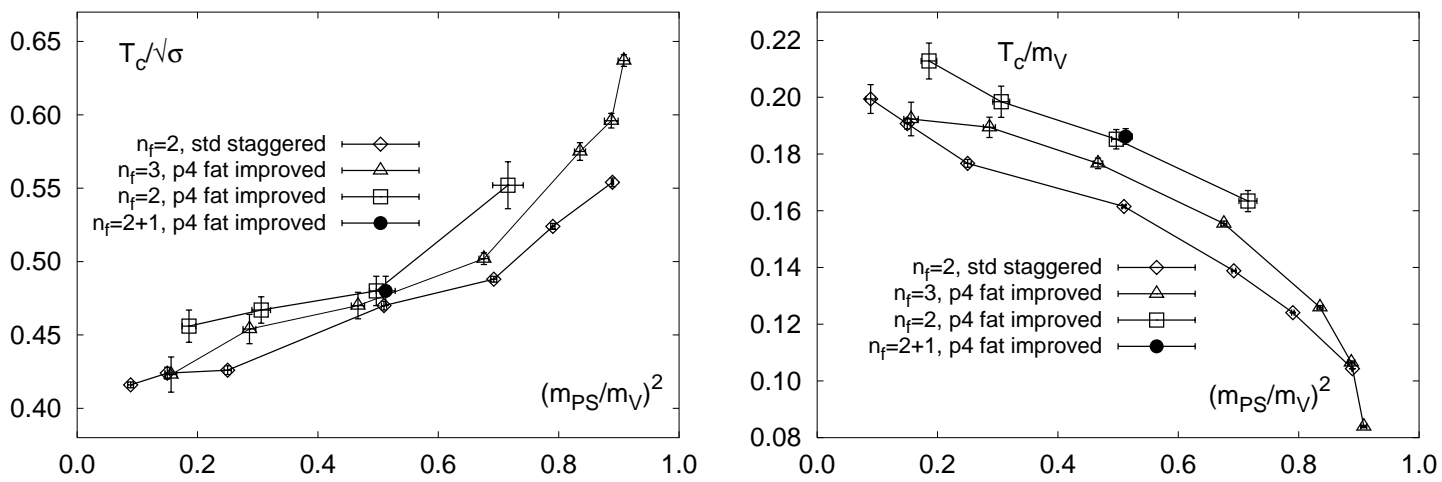

Figure 1. The critical temperature in units of $\sqrt{\sigma}$ (left) and the vector meson mass (right) versus $\left(m_{\mathrm{PS}} / m_{\mathrm{V}}\right)^{2}$. Shown are results for 2, (2+1) and 3-flavor QCD obtained from calculations with the p4 action on lattices with temporal extent $N_{\tau}=4$. For $n_{f}=2$ we also show results obtained by using unimproved gauge and staggered fermion actions.

$\beta_{c}\left(m_{q}\right)$ on lattices of size $16^{4}$. The results of this calculation are shown in Fig. 1.

We note that $T_{c} / \sqrt{\sigma}$ and $T_{c} / m_{V}$ do show a consistent flavor dependence of $T_{c}$. In a wide range of quark mass values $T_{c}$ in 3 -flavor QCD is about $10 \%$ smaller than for $n_{f}=2$. The dependence on $m_{q}$ is, however, quite different when using the vector meson mass rather than the string tension to set the scale for $T_{c}$. While $T_{c} / \sqrt{\sigma}$ does show the expected rise with increasing $m_{q}$ the contrary is the case for $T_{c} / m_{V}$. Of course, this does not come as a surprise. The vector meson mass used in Fig. 1 to set the scale is itself strongly quark mass dependent, $m_{V}=m_{\rho}+c m_{q}$. Its mass thus is significantly larger than the vector meson mass, $m_{\rho}$, in the chiral limit. We stress this well known fact here because it makes evident that one has to be careful when discussing the dependence of $T_{c}$ on parameters of the QCD Lagrangian, e.g. $n_{f}$ and $m_{q}$. One has to make sure that the observable used to set the scale for $T_{c}$ itself is not or at most only weakly dependent on the external parameters.

In Fig. 2 we show results for the ratio $\sqrt{\sigma} / m_{V}$ calculated on $16^{4}$ lattices for various values of the quark mass in 2, $(2+1)$ and 3-flavor QCD. Also shown there is the result obtained in quenched QCD as well as from a partially quenched cal- culation which we have performed on gauge field configurations generated with a dynamical (sea) quark mass of $m_{q}=0.1$. The fact that this ratio shows only little flavor dependence and that the (partially) quenched limit is in reasonable agreement with experiment and phenomenology $\beta$ indicates that the corresponding partially quenched observables are suitable also for setting the scale in the presence of dynamical quarks.

\section{1. $T_{c}$ and thermodynamics of $(2+1)$ - Flavor QCD}

Physically most relevant is an analysis of the thermodynamics of $\mathrm{QCD}$ with 2 light $\left(m_{u} \simeq\right.$ $m_{d} \simeq 0$ ) and a heavier (strange) quark with $m_{s} \simeq$ $T_{c}$. Calculations of bulk thermodynamic observables (pressure, energy density) suggest that the explicit quark mass dependence is small above $T_{c}$. In particular, a recent calculation of the pressure with $m_{s} / T=1$ and $m_{u, d} / T=0.4$ has shown that deviations from the case with three degenerate quarks are only about $10 \%$ [2]. This difference would be even smaller when $m_{s}$ rather than $m_{s} / T$ would have been kept fixed in the simulation. One thus can expect that already for $T \gtrsim 2 T_{c}$ bulk thermodynamics of the plasma phase will be well described by the equation of state of 3 degener-

\footnotetext{
${ }^{3}$ Systematic deviations are visible but are on the $10 \%$ level.
} 


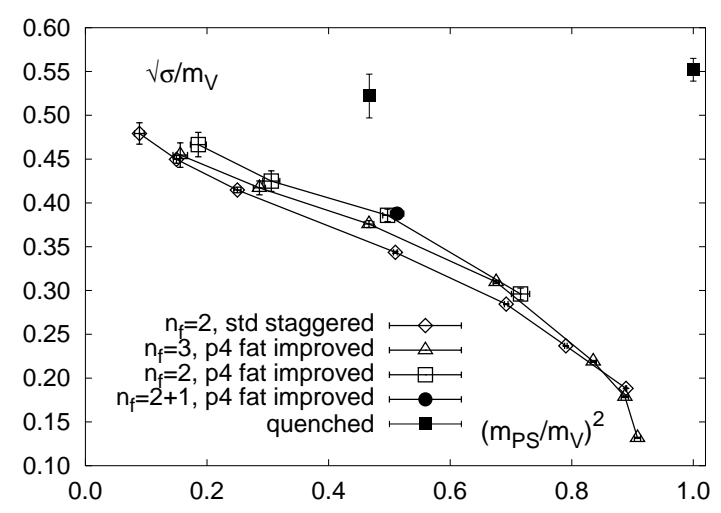

Figure 2. $\sqrt{\sigma} / m_{V}$ versus $\left(m_{\mathrm{PS}} / m_{V}\right)^{2}$ for $2,2+1$ and 3-flavor QCD obtained with the p4-action. Also shown are results obtained with unimproved staggered fermions. The filled black squares show results from quenched and partially quenched calculations with a sea quark mass of $m_{q}=0.1$.

ate, nearly massless flavors. This is different close to the transition which in the $(2+1)$-flavor case presumably is just a rapid crossover. Although this analysis has not yet been performed with a sufficiently light light quark sector the result for the pseudo-critical temperature shown in Fig. 1 suggests that $T_{c}$ is close to that of 2-flavor QCD.

\subsection{Cut-off effects}

In Fig. 1 we also show results for $T_{c}$ obtained with the standard, unimproved gauge and staggered fermion actions. We note that on lattices with temporal extent $N_{\tau}=4$ this action leads to systematically smaller values for $T_{c} / \sqrt{\sigma}$ as well as $T_{c} / m_{V}$. This provides an estimate for systematic cut-off effects, which for a wide range of quark mass values seem to be about $10 \%$. Such an estimate also is consistent with the observation that for large values of $N_{\tau}$ calculations with unimproved fermion actions do lead to larger critical temperatures. Statistical errors are, however, large in this case. We thus expect that our current analysis with improved gauge and staggered fermion actions is affected with systematic cutoff effects which are significantly below the $10 \%$ level. However, this clearly has to be checked with simulations on larger lattices.

\section{3. $\mathbf{T}_{\mathrm{c}}$ in the chiral limit}

For $n_{f}=3$ the chiral phase transition is known to be first order 泊 whereas it is most likely a continuous transition for $n_{f}=2$. This also implies that the dependence of the (pseudo)-critical temperature on the quark mass will differ in both cases. Asymptotically, i.e. to leading order in the quark mass one expects to find,

$T_{c}\left(m_{\mathrm{PS}}\right)-T_{c}(0) \sim\left\{\begin{array}{ll}m_{\mathrm{PS}}^{2 / \beta \delta} & , n_{f}=2 \\ m_{\mathrm{PS}}^{2} & , n_{f} \geq 3\end{array} \quad\right.$,

with $1 / \beta \delta=0.55$ if the 2-flavor transition indeed belongs to the universality class of $3 \mathrm{~d}, \mathrm{O}(4)$ symmetric spin models. Our estimates of $T_{c}$ in the chiral limit are based on the data shown in Fig. 1. For $n_{f}=3$ we have extrapolated $T_{c} / \sqrt{\sigma}$ and $T_{c} / m_{V}$ using an ansatz quadratic in $m_{\mathrm{PS}} / m_{V}$. In addition we have extrapolated $m_{V}$ calculated at $\beta_{c}\left(m_{q}\right)$ for $m_{q}=0.025$ and 0.05 linearly in $m_{q}$ to the critical point in the chiral limit, $\beta_{c}(0)=3.258(4)$. The extrapolation to the chiral limit is less straightforward in the case of $n_{f}=2$. The data shown in Fig. 1 indicate that the quark mass dependence for $n_{f}=2$ and 3 is quite similar. This suggests that sub-leading corrections, quadratic in $m_{\mathrm{PS}} / m_{V}$, should be taken into account in addition to the leading behavior expected from universality. In our extrapolations for $n_{f}=2$ we thus also add a term quadratic in $m_{\mathrm{PS}} / m_{V}$ to the leading term given in Eq. 1.

From these fits we estimate

$\frac{T_{c}}{m_{\rho}}=\left\{\begin{array}{ll}0.225 \pm 0.010 & , n_{f}=2 \\ 0.20 \pm 0.01 & , n_{f}=3\end{array}\right.$,

which corresponds to $T_{c}=(173 \pm 8) \mathrm{MeV}$ and $(154 \pm 8) \mathrm{MeV}$ for $n_{f}=2$ and 3, respectively. For $n_{f}=2$ this agrees well with results obtained in a calculation with improved Wilson fermions [3]. We stress, however, that the errors given here as well as in Ref. 3 are statistical only. Systematic errors resulting from remaining cut-off effects and from the ansatz used for extrapolating to the chiral limit are expected to be of similar magnitude. In order to control these errors calculations on lattices with larger temporal extent are still needed. 

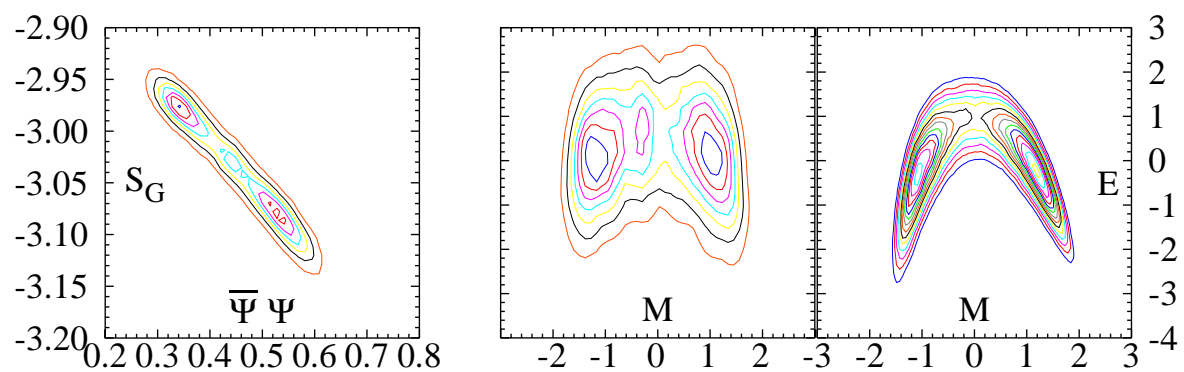

Figure 3. Contour plot for the joint probability distribution of $S_{G}$ and $\bar{\psi} \psi$ (left) as well as $E$ and $M$ (middle) for 3-flavor QCD and the 3d, 3-state Potts model (right). The QCD contour plots are based on calculations performed on a $16^{3} \times 4$ lattice at $\beta=5.1499$ with $m_{q}=0.035$. These parameters are in agreement with a previous estimate for the location of the chiral critical point [8]. The mixing parameters have been fixed to $r=0.55$ and $s=0$. Parameters for the Potts model are given in Ref. 7 .

\section{Universality at the chiral critical point of 3-flavor QCD}

The first order chiral transition of 3-flavor QCD will persist to be first order for $m_{q}>0$ up to a critical value of the quark mass. At this chiral critical point the transition will be second order and it has been conjectured that it belongs to the universality class of the $3 \mathrm{~d}$ Ising model [5]. From a simulation with standard, i.e. unimproved, gauge and staggered fermion actions we find support for this conjecture.

The analysis of the critical behavior at the $2^{\text {nd }}$ order endpoint of a line of $1^{\text {st }}$ order phase transitions requires the correct identification of energylike and ordering-field (magnetic) directions [6,7]. The ordering field at the critical point can be constructed from a linear combination of the gluonic action $S_{G}$ and the chiral condensate $\bar{\psi} \psi$,

$E=S_{G}+r \bar{\psi} \psi \quad, \quad M=\bar{\psi} \psi+s S_{G}$.

Here the mixing parameter $r$ is determined from the $m_{q}$-dependence of the line of first order transitions, $r=\left(\mathrm{d} \beta / \mathrm{d} m_{q}\right)_{\text {endpoint }}^{-1}$ and $s$ by demanding $\langle E \cdot M\rangle=0$. In fact, unlike for energy-like observables, e.g. critical amplitudes that involve the thermal exponent $y_{t}$, the universal properties of observables related to $M$ do not depend on the correct choice of the mixing parameter $s$ as long as the magnetic exponent $y_{h}$ is larger than $y_{t}$. The joint probability distribution for $E$ and $M$ characterizes the symmetry at the critical end- point and its universality class. Contour plots for the normalized $E-M$ distributions at the critical endpoints of 3-flavor QCD and the 3d, 3-state Potts model as well as the corresponding plot for the $S_{G^{-}}(\bar{\psi} \psi)$ distribution are given in Fig. 3. This shows that also in the QCD case a proper definition of energy-like and ordering-field operators is needed to reveal the symmetry properties of the chiral critical point. The joint distributions of $E$ and $M$ suggest the universal structure of the $E$ $M$ probability distribution of the 3 -d Ising model [6], although it is apparent that an analysis of 3flavor QCD on larger lattices is needed to clearly see the "two wings" characteristic for the $3 \mathrm{~d}$ Ising distribution.

\section{REFERENCES}

1. F. Karsch, Nucl. Phys. B (Proc. Suppl.) 83-84 (2000) 14.

2. F. Karsch et al., Phys. Lett. B478 (2000) 447.

3. A. Ali Khan et al. (CP-PACS Collaboration), hep-lat/0008011; S. Ejiri, these proceedings.

4. R.V. Gavai et al., Phys. Rev. Lett. 58 (1987) 2519.

5. S. Gavin et al., Phys. Rev. D49 (1994) 3079.

6. K. Rummukainen, et al., Nucl. Phys. B532 (1998) 283 and references therein.

7. F. Karsch and S. Stickan, Phys. Lett. B488 (2000) 319.

8. S. Aoki et al. (JLQCD), Nucl. Phys. B (Proc. Suppl.) 73 (1999) 459. 